Quim. Nova, Vol. 31, No. 2, 394-400, 2008

\title{
ENSAIOS PARA DETERMINAR A (BIO)DISPONIBILIDADE DE CHUMBO EM SOLOS CONTAMINADOS: REVISÃO
}

\author{
Sergio Tagliaferri Bosso e Jacinta Enzweiler* \\ Instituto de Geociências, Universidade Estadual de Campinas, CP 6152, 13083-870 Campinas SP, Brasil
}

Recebido em 2/2/07; aceito em 14/6/07; publicado na web em 26/2/08

\begin{abstract}
ESSAYS FOR DETERMINING LEAD (BIO)AVAILABILITY IN CONTAMINATED SOILS: REVIEW. Incidental ingestion of contaminated soils is a major route of $\mathrm{Pb}$ uptake by humans, especially by children. Lead speciation in soils controls its bioavailability. Bioavailability assessment requires the determination of the amount of absorbed lead if a contaminated soil is ingested. In vivo tests, which employ animals, are considered the best model to infer absorption of $\mathrm{Pb}$. But they have some logistic limitations and several authors proposed in vitro methods, which simulate conditions of human digestion. Many of them present results which correlate with in vivo essays. Several authors consider in vitro tests a good and reliable alternative to infer lead bioavailability.
\end{abstract}

Keywords: bioaccessibility; bioavailability; lead in soils.

\section{INTRODUÇÃO}

Desde a antiguidade, a mineração e o beneficiamento de minérios de chumbo para os diversos usos do chumbo metálico e de seus compostos produziram a disseminação do chumbo no ambiente. Exemplos ainda recentes são as tintas com pigmentos à base de compostos de chumbo, amplamente usadas, inclusive para a pintura de residências e o tetraetil chumbo usado como anti-detonante na gasolina durante muitas décadas. Felizmente estas duas aplicações de compostos de chumbo foram banidas na maior parte do mundo. No Brasil, elevadas concentrações de $\mathrm{Pb}$ podem ser encontradas em solos no entorno de áreas industriais, em atividades de reciclagem de baterias automotivas ou em áreas onde ocorreram atividades industriais de exploração e refino de minérios de chumbo ${ }^{1}$.

O reconhecimento dos efeitos tóxicos da exposição ao chumbo redundou na introdução de restrições ao uso de compostos de chumbo, com o objetivo de tornar o ambiente, em geral, mais seguro e saudável. O mesmo é válido para outros compostos de ação tóxica, metálicos ou não. A necessidade de monitorar a presença e o comportamento de elementos tóxicos no ambiente ensejou o desenvolvimento de métodos específicos para determinar a sua concentração e reatividade em diversas matrizes e meios.

$\mathrm{O}$ chumbo $(\mathrm{Pb})$ é um elemento-traço com abundância crustal ${ }^{2}$ de $17 \mathrm{mg} \mathrm{kg}^{-1}$ e que substitui elementos como potássio e cálcio em aluminossilicatos, em especial feldspatos, e metais em sulfetos. $\mathrm{O}$ chumbo é constituinte essencial de muitos minerais, mas somente alguns são comuns. A galena $(\mathrm{PbS})$ é o principal mineral de minério de chumbo. $\mathrm{O}$ chumbo metálico é produzido por oxidação da galena, seguida pela redução do litargírio $(\mathrm{PbO})$ formado. Alguns minerais importantes de chumbo são formados por transformação da galena. Exemplos são a reação com águas carbonatadas para formar cerussita $\left(\mathrm{PbCO}_{3}\right)$, a oxidação que produz a anglesita $\left(\mathrm{PbSO}_{4}\right)$ e a reação com fosfatos que resulta na piromorfita $\left[\left(\mathrm{Pb}_{5}\left(\mathrm{PO}_{4}\right)_{3} \mathrm{X}\right.\right.$ onde $\mathrm{X}=\mathrm{OH}^{-}, \mathrm{F}^{-}, \mathrm{Br}^{-}$ou $\left.\mathrm{Cl}^{-}\right]$.

A ocorrência desses minerais evidencia que o chumbo forma compostos de baixa solubilidade, com algumas poucas exceções (p.x., citrato e acetato). A atividade do chumbo em águas naturais, inclusive as intersticiais, com freqüência é menor que a calculada pela satura-

*e-mail: jacinta@ige.unicamp.br ção das espécies de chumbo precipitadas. Isto ocorre por causa de reações de adsorção em superfícies inorgânicas como óxidos de ferro, argilominerais, apatita e matéria orgânica. A solubilidade das diferentes espécies de chumbo, isto é, de minerais, chumbo adsorvido ou de compostos sintéticos também depende do $\mathrm{pH}$ do meio. Em $\mathrm{pH}$ ácido $(\sim 1,5)$, como o encontrado no estômago humano, muitas espécies de chumbo são solúveis, e por isto a ingestão de solos ou poeiras contaminadas pode resultar na absorção de chumbo.

O chumbo é um metal não essencial ao organismo e pode ser acumulado primeiramente em tecidos moles, e posteriormente nos $\operatorname{ossos}^{3}$. Na sua interação com organismos, o chumbo apresenta características toxicológicas comuns a outros metais (p.ex., induz anemia e danos ao rim) e também alguns efeitos específicos. A toxicidade do chumbo resulta, principalmente, de sua interferência no funcionamento das membranas celulares e enzimas, pois é capaz de formar complexos estáveis com ligantes que contêm enxofre, fósforo, nitrogênio ou oxigênio (p.ex., grupos $-\mathrm{SH},-\mathrm{H}_{2} \mathrm{PO}_{3},-\mathrm{NH}_{2},-\mathrm{OH}$ ), que funcionam como doadores de elétrons ${ }^{3}$. Por exemplo, como resultado da interação bioquímica do chumbo com grupos - $\mathrm{SH}$ de uma enzima, a atividade desta pode ser afetada e, desta forma, produzir efeitos tóxicos ${ }^{3,4}$.

Quando a exposição ao chumbo é crônica, efeitos a longo prazo no organismo podem atingir os ossos, os rins, os sistemas nervoso central, cardiovascular, reprodutivo, endócrino e a formação do feto $^{3}$. Comparadas com adultos, as crianças são mais suscetíveis aos efeitos toxicológicos do chumbo. Essa maior vulnerabilidade pode ser relacionada aos seguintes fatores ${ }^{3,5}$ : o contínuo crescimento das crianças em idade pré-escolar implica na remodelagem óssea e contribui para a transferência do chumbo dos ossos para o sangue; por terem o sistema nervoso ainda em formação, as crianças são mais vulneráveis aos efeitos neurotóxicos provocados pela contaminação por chumbo; freqüentemente, crianças apresentam deficiências metabólicas de $\mathrm{Fe}$ e $\mathrm{Ca}$, o que aumenta a absorção e os efeitos colaterais da ingestão do $\mathrm{Pb}$; a eficiência de absorção do $\mathrm{Pb}$ no trato gastro-intestinal, em crianças, é maior que em adultos, devido à formação física e ao metabolismo mais acelerado e, crianças em idade pré-escolar freqüentemente brincam diretamente na terra e têm mais acesso a poeiras e levam mãos e brinquedos sujos à boca e ao nariz, o que aumenta a possibilidade de ingestão (ou inalação) de partículas com chumbo ${ }^{6}$. 
A absorção do chumbo, ocorre no estômago (em menor escala) e no duodeno, desde que as condições sejam favoráveis. O metal deve estar solúvel, na sua forma livre $\left(\mathrm{Pb}^{2+}\right)$ ou complexado por aminoácidos, ácidos ou sais orgânicos e pelos componentes da bile, e desta forma ser transportado através da parede epitelial, onde pode ser absorvido e entrar na circulação sanguínea ${ }^{7}$. O tempo de residência do chumbo no sangue é de menos de um mês e a tendência é dele se acumular nos ossos, de onde pode ser liberado novamente.

A ingestão (ou inalação) de partículas com chumbo, sejam elas provenientes diretamente de solos ou de poeiras domésticas, é a principal via de contaminação humana, seja em crianças ou em adultos $^{8}$, exceto trabalhadores envolvidos diretamente em atividades de metalurgia, mineração, separação, refino e reciclagem de materiais e compostos de chumbo, que pela sua atividade estão mais expostos e propensos à contaminação.

Segundo Brown et al. ${ }^{9}$ o risco associado à presença de elementos tóxicos no ambiente é influenciado por três fatores: quantidade total de metal presente no ambiente; sua toxicidade e, sua biodisponibilidade. O termo biodisponibilidade oral é definido como a fração da dose administrada que atinge a circulação sanguínea a partir do trato gastro-intestinal. Portanto, a dosagem de chumbo em sangue permite avaliar se o indivíduo teve exposição recente e se absorveu o metal. Para associar estes parâmetros com a sua fonte, diversos meios, como ar, água, solos, sedimentos e alimentos, podem ser amostrados e analisados. Dentre essas matrizes, solos são analisados com grande freqüência, principalmente por métodos recomendados para tal finalidade, como os propostos pela agência de proteção ambiental americana (USA-EPA - métodos 3050B e $3051^{10}$ ). Por outro lado, já foi demonstrado que pode não haver correlação entre a quantidade total de chumbo em solos e a sua concentração no sangue de pessoas a eles expostas ${ }^{9,11}$. Uma conclusão fundamental deste tipo de estudo é que a biodisponibilidade do chumbo depende também da especiação deste elemento, isto é, das fases em que ele se encontra nos solos, e não somente da sua concentração total. A Tabela 1 apresenta uma relação entre biodisponibilidade e a forma de ocorrência de chumbo, que se refere aos minerais em que ele se encontra, tamanho de partícula, se livre ou encapsulado por minerais (p.ex., quartzo) ou materiais provenientes da metalurgia do $\mathrm{Pb}$ (escória).

Esta revisão teve como objetivo rever os métodos mais utilizados para avaliar a disponibilidade do chumbo em solos. Ênfase será dada aos ensaios que simulam o trato gastro-intestinal humano, os chamados testes in vitro ou de bioacessibilidade, que são usados para medir a quantidade de metal dissolvida se um solo contaminado é ingerido acidentalmente e, portanto, passível de absorção pelo organismo.

\section{MÉTODOS PARA ESPECIAÇÃO DE CHUMBO EM SOLOS}

A composição elementar, o estado físico e de oxidação, a fórmula empírica e a estrutura molecular são os atributos físico-químicos que caracterizam uma dada espécie química ${ }^{9}$. Nos solos, a reatividade e a solubilidade das diferentes espécies de chumbo controlam a biodisponibilidade e a toxicidade deste elemento. Por exemplo, a ordem de estabilidade dos minerais cerussita $\left(\mathrm{PbCO}_{3}\right)<$ anglesita $\left(\mathrm{PbSO}_{4}\right)<$ cloropiromorfita $\left[\mathrm{Pb}_{5}\left(\mathrm{PO}_{4}\right)_{3} \mathrm{Cl}\right]$ indica que se o chumbo estiver presente como cloro-fosfato, ele é menos solúvel e menos biodisponível $^{8}$ que como cerussita. Portanto, a avaliação de riscos associados à presença de elementos ou compostos tóxicos implica na identificação das fases em que se encontra o elemento e na estimativa das suas respectivas reatividades e mobilidades.

A associação de dados de especiação com resultados de biodisponibilidade obtidos em testes com animais (in vivo) ou ensaios laboratoriais (in vitro) demonstrou a importância da espécie na sua reatividade biológica ${ }^{8,9,11,12}$. Porém, a especiação não é o único fator controlador da solubilidade de chumbo se solos contaminados com este elemento forem acidentalmente ingeridos. Se as fases com chumbo estiverem encapsuladas por outros minerais, a cinética de reatividade destes pode ser determinante à solubilida$\mathrm{de}^{8}$. Por exemplo, a solubilidade de carbonatos e óxidos de chumbo tende a ser reduzida quando estes se encontram encapsulados por silicatos ou sulfetos ${ }^{12}$.

A caracterização das fases de chumbo em solos, às vezes, é feita por meio de difração de raios X, microssonda eletrônica e de absorção de raios X (EXAFS - "extended X-ray absorption spectroscopy"), conforme apresentam Manceau et al. ${ }^{11}$ e Davis et

Tabela 1. Tipo de ocorrência do $\mathrm{Pb}$, fórmula química e produtos de solubilidade dos minerais e a biodisponibilidade

\begin{tabular}{|c|c|c|c|}
\hline Ocorrência & Fórmula química & $\log \mathrm{K}_{\mathrm{PS}}$ & Biodisponibilidade \\
\hline \multicolumn{4}{|l|}{ Minerais } \\
\hline Anglesita & $\mathrm{PbSO}_{4}$ & 7,7 & Média \\
\hline Cerussita & $\mathrm{PbCO}_{3}$ & 12,8 & Alta \\
\hline Galena & $\mathrm{PbS}$ & $-27,5$ & Baixa \\
\hline Fluorpiromorfita & $\mathrm{Pb}_{5}(\mathrm{PO} 4)_{3} \mathrm{~F}$ & $-71,6$ & Baixa \\
\hline Hidroxipiromorfita & $\mathrm{Pb}_{3}\left(\mathrm{PO}_{4}\right)_{3} \mathrm{OH}$ & $-76,8$ & Baixa \\
\hline Cloropiromorfita & $\mathrm{Pb}_{3}\left(\mathrm{PO}_{4}\right)_{3} \mathrm{Cl}$ & $-84,4$ & Baixa \\
\hline Hinsdalita & $\mathrm{PbAl}_{3}\left(\mathrm{PO}_{4}\right)(\mathrm{OH})_{6} \mathrm{SO}_{4}$ & $-99,1$ & Baixa \\
\hline Plumbogumita & $\mathrm{PbAl}_{3}\left(\mathrm{PO}_{4}\right)_{2}(\mathrm{OH})_{5} \mathrm{H}_{2} \mathrm{O}$ & $-99,3$ & Baixa \\
\hline Corkita & $\mathrm{PbFe}_{3}\left(\mathrm{PO}_{4}\right)\left(\mathrm{SO}_{4}\right)(\mathrm{OH})_{6}$ & $-112,6$ & Baixa \\
\hline \multicolumn{4}{|l|}{ Tamanho de partícula } \\
\hline Grande & & & Baixa \\
\hline Pequena & & & Alta \\
\hline \multicolumn{4}{|l|}{ Encapsulamento* } \\
\hline Quartzo & & & Baixa \\
\hline Material amorfo (escória) & & & Média \\
\hline Óxidos de $\mathrm{Fe}-\mathrm{Pb}$ & & & Média \\
\hline $\mathrm{PbO}$ & & & Alta \\
\hline
\end{tabular}

*Composição do material que recobre partículas de $\mathrm{Pb}$. adaptado da ref. 8 
$a l{ }^{12}$. Porém, essas técnicas somente são eficientes para identificar fases de chumbo quando sua concentração é significativa e/ou se essas fases puderem ser pré-concentradas ou separadas da matriz antes das medidas ${ }^{13}$. Isto nem sempre é possível, especialmente em solos, pois várias fases diferentes podem co-existir, geralmente em concentrações individuais inferiores aos limites de detecção das técnicas empregadas. A soma de sinais característicos dificulta sua interpretação e a identificação das fases individuais onde o chumbo se encontra ${ }^{14}$.

\section{Métodos de extração seletiva}

A caracterização da reatividade química de fases metálicas associadas às frações inorgânicas e orgânicas é uma forma muito rápida e indireta de determinar a disponibilidade de metais em matrizes ambientais. As extrações químicas parciais, seqüenciais e seletivas são usadas para determinar em que fases se distribuem os diferentes elementos químicos de uma amostra, em geral de sedimentos ou solos. O método proposto por Tessier et al. ${ }^{15}$ é um dos mais citados e usados. Nele são empregadas soluções de reagentes seletivos, isto é, capazes de extrair para a fase líquida os elementos presentes em tipos específicos de fases, ou frações, por reações de troca iônica, oxi-redução e dissolução. Os reagentes, em geral, aplicados seqüencialmente à fase sólida, após a remoção da fase líquida da etapa anterior, e as frações, cuja composição é determinada são: $\mathrm{MgCl}_{2}\left(1 \mathrm{~mol} \mathrm{~L}{ }^{-1}, \mathrm{pH} 7\right)$ = íons fracamente ligados à matriz e, portanto, mais móveis, também chamada de fração trocável; NaOAc (acetato de sódio) em pH 5 = fração carbonato, à qual se associam elementos cuja solubilidade dependente do $\mathrm{pH} ; \mathrm{Na}_{2} \mathrm{~S}_{2} \mathrm{O}_{4}$ +citrato de sódio+ácido cítrico = (oxi)hidróxidos de ferro e manganês, que podem reter diversos metais e ânions por adsorção superficial e co-precipitação; $\mathrm{HNO}_{3}$ $+\mathrm{H}_{2} \mathrm{O}_{2}$, em pH 2 = matéria orgânica (p.ex.., ácidos húmicos e fúlvi-cos, microorganismos e detritos orgânicos diversos) que dentre outros mecanismos, complexa metais e, $\mathrm{HF}+\mathrm{HClO}_{4}=$ fase residual, que geralmente contêm silicatos primários e secundários (formados por intemperismo). Se esta última etapa não for efetuada sob pressão, a permanência de um pequeno resíduo pode ser devida à presença de minerais resistentes ao intemperismo e a ataques químicos (p.ex., zircão).

As extrações seqüenciais são muito utilizadas ${ }^{16} \mathrm{e}$, ao longo dos anos, variantes ao método de Tessier et al. ${ }^{15}$ foram introduzidas e alguns trabalhos de revisão reúnem a maior parte deles ${ }^{17}$. A padronização dos diferentes métodos foi proposta num protocolo harmonizado $^{18}$, que consiste somente de três etapas. A reprodutibilidade do protocolo harmonizado foi avaliada num estudo interlaboratorial apresentado por Quevauviller et al. ${ }^{19}$, e posteriormente melhorado ${ }^{20}$.

Uma das principais críticas aos métodos de extração seqüencial é a falta de seletividade das soluções extratoras usadas. Outra deficiência, apontada por Baffi et al. ${ }^{21}$, é a influência da composição mineralógica da amostra na eficiência das extrações. Isto implicaria em validar cada esquema de extração seqüencial em matriz semelhante à que se pretende estudar.

A disponibilidade de metais às vezes é avaliada pelo método TCLP"22 "Toxicity Characteristic Leaching Procedure" - método 1311), proposto pela EPA-EUA em 1994. Este método consiste em extrações parciais com ácido acético em pH 4,9 e 2,8, isto é, em condições que simulam a lixiviação realizada pela água da chuva ou por efluentes em locais contaminados. Assim como nos métodos de extração seqüencial, o TCLP é utilizado especificamente para determinar a mobilidade de analitos inorgânicos e orgânicos e avaliar seu potencial tóxico de acordo com a quantidade de contaminante que é mobilizado da matriz.

\section{Ensaios de biodisponibilidade in vivo}

Os métodos discutidos anteriormente são úteis para avaliar a distribuição de metais em fases sólidas, mas a biodisponibilidade de contaminantes é avaliada por procedimentos mais específicos, capazes de simular uma exposição ao material contaminado. Dentre eles, destacam-se os testes in vivo, que empregam cobaias (ratos, coelhos, porcos e macacos) e humanos, e são muito usados nas áreas biomédica e farmacêutica, para determinar a biodisponibilidade efetiva de medicamentos, drogas ou de materiais contaminados, ao serem administrados diretamente na dieta de cobaias ${ }^{23,24}$.

Vários testes padronizados de ensaios in vivo, para avaliar a absorção de metais pela ingestão de solos contaminados, foram sugeridos a partir de 1977, quando Dacre e Haar $^{25}$ determinaram a concentração de $\mathrm{Pb}$ em sangue e tecidos de ratos alimentados com solos contaminados por $\mathrm{Pb}$. Tais testes abrangem desde a contaminação aguda até longos períodos de exposição ${ }^{26}$. Nas cobaias, os efeitos tóxicos são avaliados por parâmetros como mortalidade, observações clínicas, exames funcionais e oftalmológicos, mudanças no peso corporal, mudanças na alimentação e no consumo de água. Já a biodisponibilidade é avaliada pela quantificação do chumbo presente no sangue, tecidos, órgãos, pêlos e ossos das cobaias ${ }^{25}$, e pelas diferenças na absorção do chumbo quando há variações na composição química e física do solo, como tamanho das partículas, forma química predominante do chumbo na matriz e quantidade administrada.

Ratos, coelhos e porcos são as cobaias usadas nos testes in vivo, no intuito de simular a ingestão de materiais contaminados por humanos. Os ratos e coelhos, devido às suas características de alimentação e pH gástrico, são considerados bons simuladores do trato gastro-intestinal infantil ${ }^{26}$, porém ensaios com porcos são os mais indicados para estimar a biodisponibilidade de $\mathrm{Pb}$ em solos. As características fisiológicas e anatômicas dos porcos, semelhantes às dos humanos, tornam sua resposta quase idêntica à de um ser humano que ingerisse o mesmo tipo de material ${ }^{27}$.

A biodisponibilidade média de chumbo presente numa dieta comum (alimentos e água) de crianças varia de 40 a $53 \%$ e em adultos de $7-15 \%$. Para a ingestão de solos e poeiras, a EPA (EUA) propõe uma biodisponibilidade média de $\mathrm{Pb}$ em torno de $30 \%$, enquanto para a água e a dieta ela é de 50\%. Estes valores encontram-se no guia do software usado para simular a contaminação de seres humanos por ingestão de solos e materiais contaminados com $\mathrm{Pb}(\text { IEUBK })^{28}$. A biodisponibilidade atribuída aos solos provém de resultados de testes in vivo executados em porcos não adultos, com solos contendo diferentes fases de $\mathrm{Pb}$, como galena, cerussita e adsorvido em (oxi)hidróxidos de $\mathrm{Fe}$ e $\mathrm{Mn}$. Esses valores são apenas aproximações, pois a biodisponibilidade também depende de aspectos sócio-econômicos da população atingida. Por exemplo, a ingestão regular de alimentos contribui para reduzir a absorção de $\mathrm{Pb}$ no trato gastro-intestinal ${ }^{29}$. A faixa etária média da população estudada também deve ser considerada, pois crianças (0-14 anos) absorvem mais $\mathrm{Pb}$ que adultos (maiores que 15 anos) ${ }^{8}$, bem como local de moradia ${ }^{30}$, que influi na acessibilidade a poeiras e solos contaminados. Estas variáveis indicam que cada área pode requerer um estudo detalhado independente ${ }^{30}$.

Um dos primeiros estudos in vivo, realizado com ratos, relacionou a biodisponibilidade com a espécie de $\mathrm{Pb}$ em solos $^{31}$. Neste estudo, os ratos foram alimentados por 30 dias com quantidades diferentes de solos contaminados por atividades de mineração do distrito industrial de Butte, em Montana (EUA). Para o cálculo de biodisponibilidade, os valores de $\mathrm{Pb}$ encontrados no sangue e tecidos dos ratos foram comparados com valores de biodisponibilidade de um grupo de cobaias alimentadas com acetato de chumbo, $\mathrm{Pb}(\mathrm{OAc})_{2}$, que é bastante solúvel. Os valores finais correspondem 
à biodisponibilidade relativa, ou seja, o total absorvido dos solos em relação ao total absorvido do $\mathrm{Pb}(\mathrm{OAc})_{2}$. Os dados permitiram avaliar a biodisponibilidade relativa média de $10 \%$ para os solos daquele distrito e de $68 \%$ para o $\mathrm{Pb}(\mathrm{OAc})_{2}$. O baixo valor de biodisponibilidade dos solos administrados aos ratos deve-se à preponderância de anglesita e galena, que são fases mais estáveis de chumbo nas condições digestivas. $\mathrm{O}$ contrário acontece com o $\mathrm{Pb}(\mathrm{OAc})_{2}$, já que sua solubilidade é elevada na faixa de $\mathrm{pH}$ encontrada nas soluções gastro-intestinais.

Davis et ll. $^{12}$ e Ruby et al. $^{24}$ realizaram ensaios em coelhos, com solos e rejeitos de mineração do mesmo distrito (Butte, MO), e os resultados de biodisponibilidade, com média de $9 \%$, são semelhantes aos de Freeman et al. ${ }^{31}$. Os resultados indicam que ratos e coelhos absorvem quantidade semelhante de $\mathrm{Pb}$ daqueles materiais e também comprovam que a composição mineralógica dos materiais contaminados controla sua biodisponibilidade.

Em outro estudo, Schoof et al. ${ }^{32}$ administraram amostras de solos e rejeitos de mineração de Sandy, Utah (EUA), além de quantidades controladas de $\mathrm{Pb}(\mathrm{OAc})_{2}$ junto à alimentação de ratos por 31 dias. Neste estudo, as amostras continham sulfato, carbonato, fosfato, arsenato e uma fase amorfa de chumbo. A biodisponibilidade relativa do $\mathrm{Pb}$ nos materiais contaminados avaliados foi, em média, três vezes maior que a encontrada por Freeman et al. ${ }^{31}$, Davis et al. ${ }^{12} \mathrm{e}$ Ruby et al. $^{24}$. Os autores ${ }^{32}$ do estudo atribuíram os valores mais elevados de biodisponibilidade à presença nas amostras de espécies mais solúveis (como carbonatos, arsenatos e a fase amorfa).

A biodisponibilidade do $\mathrm{Pb}$ em dois solos do distrito industrial de Aspen, CO (EUA), foi avaliada em porcos por Casteel et al. ${ }^{33}$. Com concentrações de 3800 e $14200 \mathrm{mg} \mathrm{kg}^{-1}$, os solos apresentaram resultados de biodisponibilidade relativa, comparados com a biodisponibilidade de $\mathrm{Pb}\left(\mathrm{OAc}_{2}\right)$, de 63 e 64\%. A alta biodisponibilidade encontrada, em comparação com métodos utilizando ratos e coelhos, é atribuída pelos autores a fatores como a fisiologia dos suínos, que excretam comparativamente menos bile que ratos e coelhos, mas aproximadamente a mesma que crianças. Outra comprovação do estudo é que a quantidade total de $\mathrm{Pb}$ não é determinante na sua biodisponibilidade. Apesar da diferença nas concentrações de $\mathrm{Pb}$ nos solos testados, os respectivos valores de biodisponibilidade tiveram uma diferença relativa de $1 \%$.

Os testes in vivo para avaliar a biodisponibilidade de $\mathrm{Pb}$ em solos contaminados são considerados confiáveis, mas eles devem ser realizados por profissionais especializados e com infra-estrutura específica, que nem sempre se encontram disponíveis. Outros fatores a considerar são os custos elevados e o tempo requerido. Esses aspectos dificultam a aplicação dos ensaios in vivo e estimularam o desenvolvimento de uma metodologia mais simplificada, rápida, relativamente confiável e barata, baseada em ensaios laboratoriais in vitro.

Sugerido por Ruby et al. ${ }^{24}$, o chamado teste de bioacessibilidade in vitro é um ensaio laboratorial que simula as condições físicoquímicas das soluções encontradas no estômago e no duodeno humanos e é usado para estimar a quantidade de $\mathrm{Pb}$ (ou outro contaminante) absorvida, caso material contaminado seja ingerido. Este tipo de teste reúne características para uma avaliação de risco rápida e confiável. A bioacessibilidade oral de uma substância foi definida como a fração solúvel nas condições do trato gastrointestinal e que está disponível para absorção ${ }^{8}$. Ainda segundo Ruby et $a .^{24}$, todo o chumbo dissolvido é passível de ser absorvido. Isto inclui o chumbo que permanece solúvel tanto na forma livre ou combinado a ligantes orgânicos pequenos, bem como também o que depois de dissolvido se liga a espécies de peso molecular elevado ou que ao entrar no duodeno é sorvido à superfície de partículas presentes.

\section{Ensaios de bioacessibilidade in vitro}

Os testes de bioacessibilidade in vitro, recentemente revisa$\operatorname{dos}^{34}$, baseiam-se na fisiologia do trato gastro-intestinal ${ }^{24}$ e simulam as condições da digestão humana. No lugar da saliva e dos sucos gástrico e duodenal naturais, são usadas soluções artificiais que simulam o meio de cada um dos compartimentos digestivos. Alguns autores (e.g. Basta e Gradwohl ${ }^{35}$ ) empregam métodos de extração seletiva como ensaios de bioacessibilidade, porém nesta revisão apenas os testes que simulam o trato digestivo humano serão considerados.

Assim como em todos os métodos discutidos acima, há vantagens e desvantagens no uso dos diversos ensaios de bioacessibilidade in vitro propostos a partir do trabalho de Ruby et al. ${ }^{24}$. A escolha de um determinado método baseia-se na sua validação, em geral, com base na comparação de resultados de testes in vivo, na fidelidade da simulação das condições fisiológicas humanas e, também, na adequação à infra-estrutura disponível. É importante ressaltar que os testes de bioacessibilidade não incluem os microorganismos presentes no trato digestivo e não consideram os mecanismos de absorção que ocorrem preferencialmente no epitélio duodenal. Uma simulação in vitro da absorção de $\mathrm{Pb}$ por parte de células do duodeno foi efetuada por Oomen et al. ${ }^{36}$, porém não será escopo desta revisão.

As soluções simuladoras do trato gastro-intestinal, empregadas nos testes de bioacessibilidade, contém enzimas, aminoácidos, sais orgânicos e inorgânicos e ácido clorídrico. Em geral, pepsina e pancreatina são as enzimas usadas. A pepsina é uma enzima ácida cuja função é metabolizar proteínas e a pancreatina, secretada no duodeno, é uma mistura de enzimas capazes de metabolizar proteínas, gorduras e carboidratos complexos ${ }^{37}$. A glicina, o aminoácido mais simples produzido pelo corpo humano, é utilizada em alguns testes e age tamponando a solução gástrica simulada, devido ao caráter anfótero da sua molécula. Alguns exemplos de sais inorgânicos utilizados são $\mathrm{NaCl}, \mathrm{KCl}, \mathrm{KSCN}$ e $\mathrm{Na}_{2} \mathrm{HPO}_{4}$, a depender do meio simulado. Os sais orgânicos são utilizados para simular a bile humana, e geralmente são empregados compostos sintéticos derivados do ácido cólico, que funcionam como emulsificantes nas soluções duodenais. Além desses reagentes, outras condições do sistema digestivo humano, como temperatura, tempo de contato entre o sólido e o biofluido e pH do meio também são reproduzidas.

Nos primeiros ensaios de bioacessibilidade, Davis et al. ${ }^{12}$ e Ruby et $a l .{ }^{24}$ estimaram a solubilidade de chumbo dos solos do distrito industrial de Butte, MT (USA), e compararam os resultados de bioacessibilidade com os resultados de biodisponibilidade obtidos em ensaios in vivo com coelhos, já mencionados. Davis et al. ${ }^{12}$ simularam as condições estomacais com uma mistura aquosa de $\mathrm{HCl}$ ( $\mathrm{pH}$ 1,3) junto com ração de coelhos rica em fibra, e o intestino com uma solução de $\mathrm{NaHCO}_{3}(\mathrm{pH} 7,0)$ e obtiveram valores baixos de solubilidade na solução intestinal, em torno de $0,18 \%$ em relação ao $\mathrm{Pb}$ total das amostras. Por outro lado, em ensaios in vivo executados paralelamente, a solubilidade média de $\mathrm{Pb}$ foi quantificada em 5,6\%. A diferença entre experimentos in vivo e in vitro foi atribuída à composição das soluções, que necessitariam conter componentes do fluído intestinal, capazes de se ligar ao $\mathrm{Pb}$ e mantê-lo em solução (p.ex., proteínas, enzimas ou ligantes orgânicos).

Ruby et al..$^{24}$ introduziram ácidos orgânicos (acético, málico e lático) na solução simuladora do fluido gástrico, enquanto na solução duodenal foram adicionados pancreatina, extratos de bile e $\mathrm{NaHCO}_{3}$. Desta forma conseguiram resultados de solubilidade in vitro mais próximos (4\%) dos obtidos nos ensaios in vivo em coelhos $(9 \%)$, realizados concomitantemente e com as mesmas amostras. Nesse trabalho ${ }^{24}$, foi empregada razão sólido/líquido de 1:10, 
que é considerada a mais próxima da quantidade de fluido gástrico produzido no estômago de coelhos, utilizados para validar o método in vitro e o $\mathrm{pH}$ inicial de 1,3 foi aumentado gradualmente na transição entre as soluções do estômago e do duodeno. Com os resultados obtidos, Ruby et al. ${ }^{24}$ demonstraram que é possível estimar a quantidade de chumbo passível de absorção, a partir da sua solubilidade em fluidos semelhantes aos do trato gastro-intestinal.

$\mathrm{O}$ mesmo teste, denominado PBET ${ }^{38}$ ("Physiologically Based Extraction Test") foi aplicado às amostras dos estudos in vivo de Freeman et $_{\text {al. }}{ }^{31}$ e Schoof et al..$^{32}$. Foram sugeridas pequenas alterações no método de Davis et al. ${ }^{12}$, como a adição da pancreatina e os sais de bile ao meio intestinal só após a solução duodenal atingir o equilíbrio em pH 7 com a adição de $\mathrm{NaHCO}_{3}$, e a razão sólido/líquido, ajustada para 1:100, para empregar a quantidade representativa de amostra $(0,4 \mathrm{~g})$ e de fluido gástrico produzido por uma criança em jejum $(40 \mathrm{~mL})$. A variação do $\mathrm{pH}$ do estômago também foi utilizada para avaliar as condições do estômago quando em jejum ( $\mathrm{pH}$ 1,3) e logo após a ingestão de alimentos ( $\mathrm{pH} 4,0)$, e também em $\mathrm{pH}$ 2,5 usado como valor médio. Os resultados foram comparáveis aos de biodisponibilidade obtidos em ratos por Freeman et al. ${ }^{31}(10 \%)$ e Schoof et al. ${ }^{32}$ (36\%), principalmente na fase gástrica e com $\mathrm{pH}$ 2,5. Para a fase intestinal, devido a maior complexidade, tanto da solução simulada quanto das condições intestinais dos ratos, os resultados apresentam as maiores diferenças relativas entre métodos e pouca reprodutibilidade. Segundo os autores, apesar da falta de precisão, essas diferenças não invalidam o teste in vitro, que apesar de superestimar a biodisponibilidade, poderia ser aplicado quando não há possibilidade de uma avaliação in vivo.

O consórcio para pesquisas em solubilidade e biodisponibilidade (SBRC), fundado em $1997^{8}$, com o objetivo de validar um método eficaz de bioacessibilidade, propôs um teste bastante simples, chamado SBET ${ }^{39}$ ("Simple Bioaccessibility Extraction Test"), uma adaptação simplificada do PBET. O SBET apenas faz a simulação da fase gástrica, com solução de ácido clorídrico e glicina, em pH 1,5. Este teste fornece resultados superestimados de bioacessibilidade, devido ao baixo $\mathrm{pH}$ do meio e à ausência de uma fase intestinal. Com este método, cerca de $90 \%$ do $\mathrm{Pb}$ total presente no material de referência certificado de solo SRM 2711 (NIST) foi dissolvido. Este resultado pode ser interpretado como a quantidade máxima de $\mathrm{Pb}$ solúvel deste material, nas condições gástricas.

Hamel et al. $^{40}$ avaliaram a influência da razão sólido/líquido nos experimentos de bioacessibilidade, com outro material de referência certificado de solo, o SRM 2710 (NIST). Ao simular apenas a fase gástrica, com uma solução simples de pepsina e $\mathrm{HCl}$ em $\mathrm{pH} 1,1$, obtiveram valores de solubilidade de chumbo entre $36 \%$ (1:100), $34 \%$ (1:1000) e $42 \%$ (1:5000) no solo certificado para as razões sólido líquido indicadas entre parênteses. Os resultados serviram como base para o desenvolvimento de um novo teste ${ }^{41}$, mais detalhado e completo, com mais duas etapas, as soluções da boca e do duodeno.

A composição da solução artificial da saliva humana usada foi a sugerida por Fusayama et $a l .{ }^{42}$ para avaliar a solubilidade de ouro e amálgama em ensaios odontológicos. A solução contém sais inorgânicos $\left(\mathrm{NaCl}, \mathrm{KCl}, \mathrm{Na}_{2} \mathrm{HPO}_{4} \mathrm{e} \mathrm{CaCl}_{2}\right)$, mucina e uréia em $\mathrm{pH}$ 5,5. Já a solução gástrica é simulada apenas com $\mathrm{HCl}$ e pepsina em $\mathrm{pH} 1,5$ e a solução duodenal contém apenas $\mathrm{Na}_{2} \mathrm{HCO}_{3}$. Os autores propõem o uso de um balanço de massas, ou seja, um protocolo de cálculos baseado nas solubilidades do $\mathrm{Pb}$ em cada etapa da extração (saliva+gástrica e saliva+gástrica+duodenal) e obtêm um valor combinado onde está expressa a massa total solúvel de $\mathrm{Pb}$ ao longo da simulação. O teste foi aplicado em amostras de solo, rejeitos de metalurgia de $\mathrm{Pb}$ e no SRM 2710. A bioacessibilidade de $\mathrm{Pb}$ em amostra de solo de Bunker Hill foi de $70 \%$ e de $62 \%$ para o SRM
2710. Estes resultados foram comparados com estudos de biodisponibilidade executados em humanos pela Columbia School of Public Health ${ }^{43}$, com a amostra de Bunker Hill, porém houve uma grande diferença entre eles, pois apenas $26 \%$, em média, do $\mathrm{Pb}$ presente na amostra é biodisponível. Essa discrepância entre os dados de ensaios in vivo e in vitro, de acordo com os autores ${ }^{41}$, poderia estar associada à absorção incompleta das espécies dissolvidas no epitélio intestinal. Esta diferença entre chumbo dissolvido e absorvido provavelmente é a principal limitação dos testes in vitro, como substitutos dos testes in vivo.

Com o mesmo método ${ }^{41}$, Ellickson et al. ${ }^{44}$ quantificaram apenas $10 \%$ do $\mathrm{Pb}$ do SRM 2710 como bioacessível. Concomitantemente, em testes realizados com ratos, foi obtida biodisponibilidade máxima de $0,9 \%$. De acordo com os autores ${ }^{44}$, essa diferença pode ser atribuída à grande quantidade de bile secretada pelos ratos, que poderia interferir na especiação e, consequientemente, na absorção do chumbo, o que também foi discutido em outros estudos ${ }^{27,31}$.

A simulação mais complexa e próxima da composição real do suco gastro-intestinal humano é apresentada por Oomem et al. ${ }^{45}$, mas ainda sem a atividade dos microorganismos presentes nas partes superior (boca+estômago) e inferior (duodeno+jejuno+íleo) do sistema digestivo humano. A saliva é simulada com pequenas quantidades de $\mathrm{KCl}, \mathrm{KSCN}, \mathrm{NaH}_{2} \mathrm{PO}_{4}, \mathrm{Na}_{2} \mathrm{HPO}_{4}, \mathrm{NaCl}, \mathrm{NaOH}$, uréia, mucina, ácido úrico e $\alpha$-amilase. $\mathrm{O}$ suco gástrico é constituído dos mesmos sais inorgânicos usados na simulação da saliva, acrescido de $\mathrm{HCl}$, glicose, ácido glucorônico, uréia, glucoseamina, albumina, pepsina e mucina. O duodeno é simulado com bile extraída de frangos, sais inorgânicos $(\mathrm{NaCl}, \mathrm{KCl}$ e $\mathrm{MgCl})$, uréia, albumina, pancreatina e lípase. A bioacessibilidade média do $\mathrm{Pb}$ presente nos solos testados foi de 54\%. Este resultado é semelhante ao obtido com soluções menos complexas.

A fração bioacessível de chumbo pela ingestão de materiais contaminados também pode ser estimada pela adição de alimentos no ensaio. Schroder et $a l .{ }^{46}$ simularam a fase gástrica com uma solução de pepsina e $\mathrm{HCl}$, em $\mathrm{pH}$ 1,8 e a fase duodenal com $\mathrm{NaHCO}_{3}$, extratos de bile e pancreatina e também extrato de proteínas para avaliar a influência do bolo alimentar na solubilidade do $\mathrm{Pb}$. Os resultados mostraram uma bioacessibilidade máxima, na simulação do organismo em jejum, de $64 \%$ (meio gástrico) e 3,2\% (meio duodenal), com médias de 36 e $1 \%$, respectivamente, em um conjunto de 18 amostras de diferentes concentrações (1270 a 14200 $\left.\mathrm{mg} \mathrm{kg}^{-1}\right)$. A adição do extrato de proteínas reduziu a quantidade média de $\mathrm{Pb}$ solúvel na etapa gástrica para $23 \%$, com valor máximo de $36 \%$. Estes resultados foram comparados, no mesmo estudo, com os obtidos em ensaios in vivo em porcos, aos quais foi administrada a mesma alimentação e os mesmos solos. Os resultados dos dois ensaios apresentaram correlação forte, exceto os da fase duodenal na ausência do extrato de proteínas. Os autores concluíram que o ensaio in vitro pode ser usado para estimar a biodisponibilidade relativa de $\mathrm{Pb}$, As e $\mathrm{Cd}$ em solos.

A bioacessibilidade do $\mathrm{Pb}$ em poeiras oriundas de solos contaminados também pode ser avaliada com testes in vitro. Yu et al. ${ }^{47}$ aspiraram poeiras de carpetes de residências próximas a um distrito industrial de New Jersey (EUA) e usaram o método proposto por Hamel et $a l .{ }^{41}$. Os resultados indicam elevada solubilidade relativa de chumbo nessas poeiras, com valor de até $77 \%$ (média de $64 \%$ ) na solução gástrica e de até $32 \%$ (média de 12\%) na solução duodenal. Os dados de bioacessibilidade foram aplicados ao modelo computacional IEUBK ${ }^{28}$ ("Integrated Exposure Uptake Biokinetic Model for Lead in Children"). Os resultados indicaram que há correlação entre os dados de bioacessibilidade do $\mathrm{Pb}$ obtidos com a simulação gástrica e os níveis de $\mathrm{Pb}$ no sangue de crianças expostas às poeiras.

Além dos métodos discutidos e revisados neste trabalho, ou- 
Tabela 2. Principais métodos de bioacessibilidade, tipo de experimento, constituintes das soluções simuladas, $\mathrm{pH}$ e tempo de contato de cada simulação. Todos testes são executados em temperatura controlada $\left(37^{\circ} \mathrm{C}\right)$, na proporção sólido:líquido $(\mathrm{S} / \mathrm{L})$ indicada. $\mathrm{O}$ último (TIM) é realizado em fluxo

\begin{tabular}{|c|c|c|c|c|c|}
\hline Método & Solução simulada & $\mathrm{pH}$ & $\mathrm{S} / \mathrm{L}$ & Tempo (h) & Ref. \\
\hline SBET & Gástrica - Glicina & 1,5 & $1 / 100$ & 1 & 39 \\
\hline \multirow[t]{2}{*}{ PBET } & $\begin{array}{c}\text { Gástrica - Pepsina, ac. málico, ac. acético, ac. } \\
\text { lático e ac. cítrico }\end{array}$ & 2,5 & $1 / 100$ & 1 & 38 \\
\hline & Intestinal - Pancreatina, sais de bile e $\mathrm{NaHCO}_{3}$ & 7,0 & & 3 & \\
\hline \multirow[t]{2}{*}{ IVG } & Gástrica - Pepsina e $\mathrm{NaCl}$ & 1,8 & $1 / 150$ & 1 & 46 \\
\hline & Intestinal - Pancreatina, extrato de bile e $\mathrm{NaHCO}_{3}$ & 5,5 & & 1 & \\
\hline U.S.P & Gástrica - Pepsina e $\mathrm{NaCl}$ & 1,2 & $1 / 100$ & 2 & 40 \\
\hline \multirow[t]{3}{*}{ MB \& SR } & Saliva - Mucina, uréia, $\mathrm{Na}_{2} \mathrm{HPO}_{4}, \mathrm{CaCl}_{2}, \mathrm{KCl}, \mathrm{NaCl}$ & 5,5 & $1 / 160$ & $5 \mathrm{~s}$ & 41 \\
\hline & Gástrica - Pepsina e $\mathrm{NaCl}$ & 1,5 & $1 / 2160$ & 2 & \\
\hline & Intestinal $-\mathrm{NaHCO}_{3}$ & 7,0 & $1 / 4770$ & 4 & \\
\hline \multirow[t]{2}{*}{ DIN } & Gástrica - Pepsina, mucina & 2,0 & $1 / 15$ & 2 & 48 \\
\hline & Intestinal - Tripsina, pancreatina e extrato de bile & 7,5 & $\begin{array}{c}1 / 50 \\
1 / 100\end{array}$ & 6 & \\
\hline \multirow[t]{2}{*}{ SHIME } & $\begin{array}{l}\text { Gástrica - Pectina, Nutrilon plus, mucina, amido, } \\
\text { cellobiose, glucose, proteose peptona e leite }\end{array}$ & 5,2 & $1 / 2,5$ & 3 & 39 \\
\hline & Intestinal - Pancreatina, blie bovina e $\mathrm{NaHCO}_{3}$ & 6,5 & $1 / 4$ & 5 & \\
\hline \multirow[t]{3}{*}{ RIVM } & $\begin{array}{c}\text { Saliva - Mucina, amilase, uréia, ac. úrico, } \mathrm{NaOH} \text {, } \\
\qquad \mathrm{NaCl}, \mathrm{KCl}, \mathrm{Na}_{2} \mathrm{SO}_{4}, \mathrm{NaSCN}\end{array}$ & 6,5 & $1 / 15$ & $5 \mathrm{~min}$ & 39 \\
\hline & $\begin{array}{c}\text { Gástrica - Pepsina, glucose, ac. glucorônico, } \\
\text { glucoseamina, BSA, mucina }\end{array}$ & 1,1 & 1,40 & 2 & \\
\hline & $\begin{array}{c}\text { Intestinal - Lípase, pancreatina, uréia, BSA e } \\
\text { extratos de bile }\end{array}$ & 5,5 & $1 / 100$ & 2 & \\
\hline \multirow[t]{3}{*}{ TIM } & Saliva - Não especificado & 5,0 & $1 / 5$ & $5 \mathrm{~min}$ & 39 \\
\hline & Gástrica - Lípase, pepsina & 2,0 & $1 / 30$ & 1,5 & \\
\hline & Intestinal - Pancreatina, extrato de bile, $\mathrm{NaHCO}_{3}$ & 7,2 & $1 / 50$ & 6 & \\
\hline
\end{tabular}

SBET- Simple Extraction Bioaccessibility Test; PBET- Physiologically Based Extraction Test; IVG- In vitro Gastrointestinal model; U.S.PU.S. Pharmacopoeia gastric model; MB \& SR- Mass Balance and Soil Recapture; DIN- Static gastrointestinal model; SHIME- Simulator of Human Intestinal Microbial Ecosystems of Infants; RIVM- In Vitro Digestion model; TIM- Dynamic Gastrointestinal model.

Tabela 3. Comparação de resultados de bioacessibilidade de $\mathrm{Pb}$ (valores mínimos e máximos) obtidos em solos contaminados, suas respectivas concentrações e método utilizado

\begin{tabular}{|c|c|c|c|c|c|}
\hline Tipo de amostra & $\begin{array}{l}\text { Concentração } \\
\left(\mathrm{mg} \mathrm{kg}^{-1}\right)\end{array}$ & Método & Fluido simulado & $\begin{array}{c}\mathrm{Pb} \text { bioacessível } \\
(\%)\end{array}$ & Ref. \\
\hline Solo (poeiras) & 2600 & PBET & Gástrico & 77 & 49 \\
\hline Solos & $1600-16200$ & PBET & Gástrico & $11-37$ & 50 \\
\hline Solos & $400-4000$ & PBET & Gástrico + Duodenal & $13-64$ & 51 \\
\hline Solos & $150-4800$ & SBET & Gástrico + Duodenal ${ }^{*}$ & $0,5-85$ & 52 \\
\hline Solos & $4000-7000$ & W-PBET ${ }^{* *}$ & Gástrico + Duodenal & $12-27$ & 53 \\
\hline Solos & $215-1580$ & SBET & Gástrico & $46-50$ & 54 \\
\hline Solos & $500-5000$ & Hamel $^{40,41}$ & Gástrico & $50-80$ & 55 \\
\hline
\end{tabular}

"Simulação duodenal feita com $\mathrm{NaHCO}_{3}$; ${ }^{* *}$ PBET adaptado para simulação do trato gastro-intestinal de gansos

tros podem ser encontrados na literatura. A Tabela 2 resume alguns destes trabalhos, com o nome do método, as soluções empregadas e as condições experimentais de cada um deles. Alguns testes, como o DIN e o SHIME, também avaliam o efeito da adição de alimentos (leite, proteínas ou amido) na bioacessibilidade do $\mathrm{Pb}$, simulando as condições gastro-intestinais após a alimentação.

A Tabela 3 apresenta uma comparação dos resultados de bioacessibilidade de $\mathrm{Pb}$, em solos, apresentados na literatura. Os trabalhos podem apresentar algumas diferenças quanto à execução do método, como o tipo de agitação da solução+amostra, separação das frações e técnica analítica usada para determinar o chumbo na fração solúvel mas, de modo geral, apresentam os princípios dos métodos originais.

\section{CONCLUSÕES}

A presença de materiais contaminados em locais habitados ou remotos é indesejável à biota. Mas com frequiência a sociedade se depara com a presença de concentrações anômalas e elevadas de elementos tóxicos em locais habitados. A origem destas anomalias pode ser geológica, mas muitas vezes é antrópica e, em geral, o risco associado deve ser avaliado. No caso do chumbo, bem como de muitos outros elementos-traço, a presença de elevadas concentrações em solos, por si só não é suficiente para tirar conclusões, pois a biodisponibilidade depende da sua especiação na matriz considerada.

A determinação da(s) fase(s) em que se encontra o chumbo idealmente é feita por meio de técnicas instrumentais, mas muitas 
vezes a concentração individual das fases não é suficiente para a identificação, e a mistura de fases também pode adicionar dificuldades adicionais à caracterização. Apesar disto, a avaliação de risco objetiva responder se a ingestão acidental dos solos ou de suas poeiras implica em absorção do chumbo pelo organismo humano, especialmente por crianças, que são mais suscetíveis. Extrações sequienciais podem ser úteis na avaliação da solubilidade das frações com chumbo, mas não respondem à questão da solubilidade em condições gastro-intestinais.

Para efeito de avaliação de risco, os órgãos gestores do meio ambiente empregam dados de biodisponibilidade realizados com cobaias. Mas os testes in vivo, pelas suas características, requerem infra-estrutura e pessoal especializado, demandam tempo e são caros. Para superar essas limitações, vários ensaios de bioacessibilidade ou testes in vitro, executados em condições que tentam simular as condições físico-químicas do trato gastro-intestinal humano, foram propostos, testados e muitas vezes validados por comparação com resultados de testes in vivo. Os resultados destes ensaios são reprodutíveis e apesar de muitas vezes superestimarem a biodisponibilidade, são úteis porque a estimam de maneira confiável e rápida. Os testes de bioacessibilidade in vitro despertam grande interesse, empregam materiais e reagentes facilmente encontrados e possibilitam a avaliação de muitas amostras simultaneamente. Apesar disto, vários aspectos ainda devem ser melhorados, principalmente no que se refere às diferenças com os resultados de ensaios in vivo, a fim de limitar o uso destes.

\section{REFERÊNCIAS}

1. Paoliello, M. M. B.; De Capitani, E. M.; Rev. Environ. Contam. T. 2005, $184,59$.

2. Wedepohl, K. H.; Geochim. Cosmochim. Acta 1995, 59, 1217.

3. Moreira, F. R.; Moreira, J. C.; Pan American Journal of Public Health 2004, 15, 119; Barbosa Jr., F.; Tanus-Santos, J. E.; Gerlach, R. F.; Parsons, P. J.; Environ. Health Persp. 2005, 113, 1669.

4. Strydom, C.; Robinson, C.; Pretorius, E.; Whitcutt, J. M.; Marxand, J.; Bornman, M. S.; Water SA. 2006, 32, 543.

5. Claudio, E. S.; Goldwin H. A.; Magyar J. S.; Prog. Inorg. Chem. 2003, 51,1 .

6. Koller, K.; Brown T.; Spurgeon J.; Levy, L.; Environ. Health Persp. 2004, $112,987$.

7. Oomen, A. G.; Tolls, J.; Sips, A. J. A. M.; Groten, J. P.; Arch. Environ. Contam. Toxicol. 2003, 44, 116.

8. Ruby, M. V.; Schoof, R.; Brattin, W.; Goldade, M.; Post, G.; Harnois, M.; Mosby, D. E.; Casteel, S. W.; Berti, W.; Carpenter, M.; Edwards, D.; Cragin, D. W.; Chappell, W.; Environ. Sci. Technol. 1999, 33, 3697.

9. Brown, G. E. Jr; Foster, A. L.; Ostergren, E.; Proc. Natl. Acad. Sci. U.S.A. 1999, 96, 3388

10. http://www.epa.gov/epaoswer/hazwaste/test/3_series.htm, acessada em Dezembro 2006.

11. Manceau, A.; Boisset M.; Sarret, G.; Hazemann, J.; Mench, M.; Cambier, P.; Prost, R.; Environ. Sci. Technol. 1996, 30, 1540.

12. Davis, A.; Ruby, M. V.; Bergstrom, P. D.; Environ. Sci. Technol. 1992, $26,461$.

13. Ostergren, J. D.; Brown, G. E. Jr; Parks, G. A.; Tingle, T. E.; Environ. Sci. Technol. 1999, 33, 1627.

14. Bargar, J. R.; Towle, S. N.; Brown, G. E. Jr; Parks, G. A.; J. Colloid Interface Sci. 1997, 185, 473.

15. Tessier, A.; Campbell, P. G. C.; Bisson, M.; Anal. Chem. 1979, 51, 844.

16. Kim, N. D.; Fergusson J. E.; Sci. Total Environ. 1990, 105, 191; Sims, J. T.; Kline, J. S.; J. Environ. Qual. 1991, 20, 387; Sheppard, M. I.; Thibault D. H.; Soil Sci. Soc. Am. J. 1992, 56, 415.

17. Gleyzes, C.; Tellier, S.; Astruc, M.; Trends Anal. Chem. 2002, 21, 461; Hlavay, J.; Prohaska, T.; Weisz, M.; Wenzel, W. W.; Stingeder, G. J.; Pure Appl. Chem. 2004, 76, 415.

18. Ure, A.; Quevauviller, P.; Muntau, H.; Griepink, B.; Int. J. Environ. Anal. Chem. 1993, 51, 135.
19. Quevauviller, P.; Rauret, G.; Muntau, H.; Ure, A. M.; Rubio, R.; LopezSanchez, J. F.; Fiedler, H. D.; Griepink, B.; Fresenius J. Anal. Chem. 1994, 349, 808.

20. Rauret, G.; Lopez-Sanchez, J. F.; Sahuquillo, A.; Rubio, R.; Davidson, C.; Ure, A.; Quevauviller, P.; J. Environ. Monit. 1999, 1, 57.

21. Baffi, F.; Ianni, C.; Ravera, M.; Soggia, F.; Magi, E.; Anal. Chem. Acta 1998, 360, 27.

22. Environmental Protection Agency, USA; Method 1311 - Toxicity Characteristic Leaching Procedure, 1994.

23. Sandstea, H. H.; P. Soc. Exp. Biol. Med. 1967, 124, 18

24. Ruby, M. V.; Davis, A.; Link, T. E.; Schoof, R.; Chaney, R. L.; Freeman, G. B.; Bergstrom, P.; Environ. Sci. Technol. 1993, 27, 2870.

25. Dacre, J. C.; Haar, T.; Environ. Contaminants Toxicol. 1977, 6, 11.

26. Organisation for Economic Co-operation and Development; OECD Guideline 407, 1995; OECD Guideline 408, 1998; OECD Guideline 409, 1998.

27. Hettiarachchi, G. M.; Pierzynski, G. M.; Environ. Prog. 2004, 23, 78.

28. Environmental Protection Agency, USA; User's guide for the integrated exposure uptake biokinetic model for lead in children (IEUBK), 2002.

29. Heard, M. J.; Chamberlain, A. C.; Sci. Total Environ. 1983, 30, 245.

30. Lanphear, B. P.; Matte, T. D.; Rogers, J.; Clickner, R. P.; Dietz, B.; Bornschein, R. L.; Succop, P; Mahaffey, K. R.;Dixon, S.; Galke, W.; Rabinowitz, M.; Farfel, M.; Rohde, C.; Schwartz, J.; Ashley, P.; Jacobs, D. E.; Environ. Res. 1998, 79, 51.

31. Freeman, G. B.; Johnson, J. D.; Killinger, J. M.; Liao, S. C.; Feder, P. I.; Davis, A. O.; Ruby, M. V.; Chaney, R. L.; Lovre, S. C.; Bergstrom, P. D.; Fund. Appl. Toxicol. 1992, 19, 388.

32. Schoof, R. A.; Butcher, M. K.; Sellstone, C.; Ball, R. W.; Fricke, J. R.; Keller, V.; Keen, B.; Environ. Geochem. Health 1995, 17, 189.

33. Casteel, S. W.; Cowart, R. P.; Weis, C. P.; Henningsen, G. M.; Hoffman, E.; Brattin, W. J.; Guzman, R. E.; Starost, M. F.; Payne, J. T.; Stockham, S. L.; Becker, S. V.; Drexler, J. W.; Turk, J. R.; Fundam. Appl. Toxicol. 1997, 36, 177.

34. Intawongse, M.; Dean, J. R.; TrAC, Trends Anal. Chem. 2006, 25, 876.

35. Basta, N.; Gradwohl, R.; J. Soil Contam. 2000, 9, 149.

36. Oomen, A. G.; Tolls, J.; Sips, A. J. A. M.; van den Hoop, M. A. G. T.; Arch. Environ. Contam. Toxicol. 2003, 44, 107.

37. Guyton, A. C.; Hall, J. E.; Tratado de Fisiologia Médica, $11^{\text {th }}$. ed., Elsevier: Rio de Janeiro, 2006.

38. Ruby, M. V.; Davis, A.; Schoof, R.; Eberle, S.; Sellstone, C. M.; Environ. Sci. Technol. 1996, 30, 422.

39. Oomen, A. G.; Hack, A.; Minekus, M.; Zeijdner, E.; Cornelis, S. C.; Schoeters G.; Verstraete, W.; van de Wiele, T.; Wragg, J.; Rompelberg, C. J. M.; Sips, A.; van Wijnen J. H.; Environ. Sci. Technol. 2002, 36, 3326.

40. Hamel, S. C.; Buckeley, B.; Lioy, P. J.; Environ. Sci. Technol. 1998, 32, 358.

41. Hamel, S. C.; Ellickson, K. M.; Lioy, P. J.; Sci. Total Environ. 1999, 244, 273.

42. Fusayama, T.; Nomoto, S.; Katayori, T.; J. Dent. Res. 1963, 42, 1183.

43. Maddaloni, M.; Lolacono, N.; Manton, W.; Blum, C.; Drexler, J.; Graziano, J.; Environ. Health Perspect. 1998, 106 (Suppl 6), 1589.

44. Ellickson, K. M.; Meeker, R. J.; Gallo, M. A.; Buckley, B. T.; Lioy, P. J.; Arch. Environ. Contam. Toxicol. 2001, 40, 128.

45. Oomen, A. G.; Rompelberg, C. J. M.; Bruil, M. A.; Dobbe, C. J. G.; Pereboom, D. P. K. H.; Sips, A. J. A. M.; Arch. Environ. Contam. Toxicol. 2003, 44, 281.

46. Schroder, J. L.; Basta, N. T.; Casteel, S. W.; Evans, T. J.; Payton, M. E.; Si, J.; J. Environ. Qual. 2004, 33, 513.

47. Yu, C. H.; Yiin, L.; Lioy, P. J.; Risk Analysis 2006, 1, 125.

48. Hack, A.; Selenka, F.; Toxicol. Lett. 1996, 88, 199.

49. Sonmez, O.; Pierzynski, G. M.; Water, Air, Soil Pollut. 2005, 166, 3.

50. Carrizales, L.; Razo, I.; Tellez-Hernandez, J. I.; Torres-Nerioa, R.; Torres, A.; Batres, L. E.; Cubillas, A. C.; D1az-Barriga, F.; Environ. Res. 2006, $101,1$.

51. Lee, S. W.; Lee, B. T.; Kim, J. Y.; Kim, K. W.; Lee, J. S.; Environ. Mont. Assess. 2006, 119, 233.

52. Navarro, M. C.; Perez-Sirvent, C.; Mart1nez-Sanchez, M. J.; Vidal, J.; Marimon, J.; Chemosphere 2006, 63, 484.

53. Furman, O.; Strawn, D. G.; Heinz, G. H.; Willians, B.; J. Environ. Qual. 2006, 35,450

54. Edwin F.; Barth, E. F.; Succop, P. A.; Evans, M. L.; Environ. Mont. Assess. 2005, 110, 257.

55. Kientz, K.; Jimenez, B. D.; Perez, L.; Rodr1guez-Sierra, C. J.; Bull. Environ. Contam. Toxicol. 2003, 70, 927. 